\title{
Ant venom immunotherapy in Australia: the unmet need
}

Raymond J Mullins PhD, FRACP, FRCPA Consultant Physician Immunology and Allergy

Simon GA Brown MBBS, PhD, FACEM Professor in Emergency Medicine, ${ }^{2}$ and Head ${ }^{3}$

1 John James Medical Centre, Canberra, ACT.

2 University of Western Australia and Royal Perth Hospital, Perth, WA.

3 Centre for Clinical Research in Emergency Medicine, Harry Perkins Institute of Medical Research, Perth, WA.

simon.brown@ uwa.edu.au ack jumper ant (JJA) venom extract is available through the Therapeutic Goods Administration (TGA) Special Access Scheme, and it is now time to provide this treatment alongside other insect venom immunotherapies (VITs) to allergic patients in areas of Australia where JJAs are prevalent.

The risk of insect sting anaphylaxis depends on complex interactions between the likelihood of human contact, insect aggression and regional distribution of the causative insect. Whereas venom allergy prevalence and presentation rates may be relatively low in highly urbanised areas, in rural areas and highly exposed populations, the prevalence of previous systemic reactions to stings can approach $3 \%-4 \%, 1,2$ and up to $30 \%$ of cases of anaphylaxis presenting to emergency departments (EDs) are triggered by insect stings. ${ }^{3}$ There were 20 deaths attributed to insect stings in Australia between 1997 and 2005. ${ }^{4}$

The Australian JJA (Myrmecia pilosula) (Box) is an aggressive species with patchy distribution around Australia, causing severe anaphylaxis, mainly in the south-eastern states. ${ }^{5}$ A Tasmanian allergist, Paul Clarke, first highlighted the extent of this problem in the Journal in $1986,{ }^{6}$ and the first detailed description of four JJA sting anaphylaxis deaths was published in $2000 . .^{7}$ In Tasmania, the prevalence of JJA venom allergy is around $2.7 \%$, and the rate of ED presentation with anaphylaxis for JJA is twice that for honeybees. ${ }^{1,3}$ Attempts to desensitise JJA-allergic patients with a crushed whole-body preparation produced by CSL were ineffective and that product was withdrawn in the 1990s. ${ }^{8}$

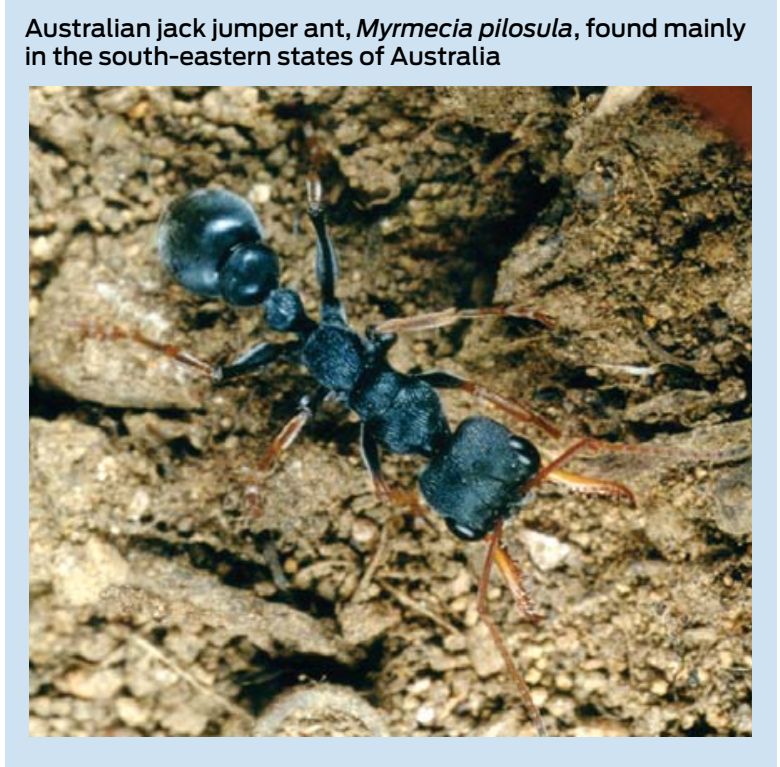

\section{Summary}

Jack jumper ant (JJA) venom allergy is an important cause of anaphylaxis in south-eastern Australia.

- The efficacy and real-world effectiveness of JJA venom immunotherapy (VIT) to prevent anaphylaxis in allergic patients are now well established, with an evidence base that is at least equivalent to that supporting VIT for allergy to other insect species.

The tolerability and safety of JJA VIT are comparable with those of honeybee VIT.

Management of sting anaphylaxis centres on identifying the causative insect, avoidance strategies where possible (eg, nest removal, moving to a location where the species is absent or less common), providing an emergency action plan and specific VIT. Although cost-effectiveness is difficult to quantify, ${ }^{9}$ VIT for wasps and honeybees is a well established procedure associated with lower rates of recurrent anaphylaxis and higher quality of life compared with carrying an adrenalin autoinjector alone. ${ }^{10}$ The efficacy of VIT directed against JJA has been established recently in a randomised, double-blind, placebo-controlled trial. ${ }^{11}$ The method was further refined in another randomised comparison of different treatment regimens, ${ }^{12}$ and a diagnostic framework has been developed to identify suitable candidates for treatment with this extract in mainland Australia as well as in Tasmania. ${ }^{5}$ Real-world efficacy is also being monitored; treatment tolerability and compliance are comparable with honeybee VIT, and in 478 patients receiving JJA VIT between 2002 and 2012, six of 132 individuals who experienced accidental stings (4.5\%) had systemic allergic reactions, and only one individual was given adrenalin (own unpublished data).

Purified JJA venom for VIT is not commercially available, but is prepared by the Tasmanian Jack Jumper Allergy Program at the Royal Hobart Hospital as an active pharmaceutical ingredient (ie, concentrate), and use requires prior approval through the TGA Special Access Scheme. Although the evidence base supporting JJA VIT is at least equivalent to that supporting VIT for the honeybee, European wasp and paper wasp, which is subsidised through the Pharmaceutical Benefits Scheme, there is no equivalent subsidy for JJA venom supplies and the cost must be covered in full by the hospitals and/or patients. The cost of supplying JJA venom extract currently ranges from $\$ 1000$ to $\$ 3000$ per patient per year, depending on the dose schedule. This is more than for other insects because of the relatively small market volume. On the basis of our experience in Tasmania with enrolment rates, dropouts and treatment completion, we expect that subsidising the 
treatment nationally would result in a "steady state" of 500 to 1000 patients receiving treatment at any one time.

In response to this issue, the Australasian Society for Clinical Immunology and Allergy (ASCIA) has released a literature review and position statement on ant venom immunotherapy in Australia. ${ }^{13}$ ASCIA recognises that JJA VIT is unlikely to generate commercial venom development and, in the interest of equity, calls on federal and state governments to recognise the clinical need for this uniquely Australian problem and to allocate specific funding for hospital clinical services to provide this treatment. The statement also recognises the need for funding for ongoing research to investigate the use of lower dose protocols, immunological adjuvants and pharmaceutical research to investigate more stable formulations for distribution and supply to clinicians. However, ASCIA's key messages are that the evidence base supporting JJA VIT now matches if not exceeds the evidence base supporting VIT for other species, and so it is now time to provide this treatment alongside other insect VITs to allergic patients in areas of Australia where JJAs are prevalent.

Acknowledgements: Simon Brown is supported by National Health and Medical Research Council Career Development Fellowship Award ID1023265.

Competing interests: Simon Brown has led the research program developing JJA VIT and is employed as a consultant to the Jack Jumper Allergy Program at the Royal Hobart Hospital, the supplier of venom extract for clinical use.

Provenance: Not commissioned; externally peer reviewed.

1 Brown SGA, Franks RW, Baldo BA, Heddle RJ. Prevalence, severity, and natural history of jack jumper ant venom allergy in Tasmania. J Allergy Clin Immunol 2003; 111: 187-192.
2 Douglas RG, Weiner JM, Abramson MJ, O'Hehir RE. Prevalence of severe ant-venom allergy in southeastern Australia. J Allergy Clin Immunol 1998; 101 (1 Pt 1): 129-131.

3 Brown SGA. Clinical features and severity grading of anaphylaxis. J Allergy Clin Immunol 2004; 114: 371-376.

4 Liew WK, Williamson E, Tang ML. Anaphylaxis fatalities and admissions in Australia. J Allergy Clin Immunol 2009; 123: 434-442.

5 Brown SGA, van Eeden P, Wiese MD, et al. Causes of ant sting anaphylaxis in Australia: the Australian Ant Venom Allergy Study. Med J Aust 2011; 195: 69-73.

6 Clarke PS. The natural history of sensitivity to jack jumper ants (Hymenoptera formicidae Myrmecia pilosula) in Tasmania. Med J Aust 1986; 145: 564-566.

7 Brown SGA, Wu QX, Kelsall GR, et al. Fatal anaphylaxis following jack jumper ant sting in southern Tasmania. Med J Aust 2001; 175: 644-647.

8 Weiner JM, Baldo BA, Donovan GR, Sutherland SK. Allergy to jumper ant (Myrmecia pilosula) stings in south-eastern Australia. Ann Allergy Asthma Immunol 1995; 74: 60.

9 Ruëff F, Biló MB, Cichocka-Jarosz E, et al. Immunotherapy for hymenoptera venom allergy: too expensive for European health care? Allergy 2013; 68 : 407-408.

10 Oude Elberink JN, van der Heide S, Guyatt GH, Dubois AE. Immunotherapy improves health-related quality of life of adult patients with dermal reactions following yellow jacket stings. Clin Exp Allergy 2009; 39 : 883-889.

11 Brown SGA, Wiese MD, Blackman KE, Heddle RJ. Ant venom immunotherapy: a double-blind, placebo-controlled, crossover trial. Lancet 2003; 361: 1001-1006.

12 Brown SGA, Wiese MD, van Eeden P, et al. Ultrarush versus semirush initiation of insect venom immunotherapy: a randomized controlled trial. J Allergy Clin Immunol 2012; 130: 162-168.

13 Australasian Society for Clinical Immunology and Allergy. Position statement: jack jumper ant venom immunotherapy. 2013. http://www. allergy.org.au/health-professionals/papers/jack-jumper-ant-venomimmunotherapy (accessed Oct 2013). 